\section{Desarrollo y validación de encuesta de percepción del portafolio en estudiantes de medicina de pregrado}

\author{
ARNOLDO RIQUELME ${ }^{1,2,3,7}$, BENJAMÍN MÉNDEZ \\ PALOMA DE LA FUENTE ${ }^{4, a}$, OSLANDO PADILLA ${ }^{5}$, CARLA BENAGLIO $^{6}$, \\ MARISOL SIRHAN ${ }^{1,3}$, JAIME LABARCA $^{7}$

\section{Development and validation of a questionnaire on perception of portfolio by undergraduate medical students}

Background: Portfolio is an innovative instrument that promotes reflection, creativity and professionalism among students. Aim: To describe the development and validation process of a questionnaire to evaluate the use of portfolio in undergraduate medical students. Material and Methods: Focus groups with students and teachers were employed to identify aspects related with portfolio in undergraduate teaching. The Delphi technique was used to prioritize relevant aspects and construct the questionnaire. The validated questionnaire, consisting in 43 items and 6 factors, was applied to 97 students (response rate of 99.9\%) in 2007 and 100 students (99.2\%) in 2008. Each question had to be answered using a Likert scale, from 0 (completely disagree) to 4 (completely agree) The validity and reliability of the questionnaire was evaluated. Results: The questionnaire showed a high reliability (Cronbach alpha $=0.9)$. The mean total scores obtained in 2007 and 2008 were $106.2 \pm 21.2$ (61.7\% of the maximal obtainable score) and $104.6 \pm 34.0$ (60.8\% of the maximal obtainable score), respectively. No significant differences were seen in the analysis by factors. Changes in portfolio during 2008 showed differences in items related with organization, evaluation and regulation. Conclusions: The questionnaire is a valid and highly reliable instrument, measuring perceptions about the portfolio by undergraduate medical students. The students perceived an improvement in their creativity and professionalism as one of the strengths of portfolio. The weaknesses identified during the implementation process helped us to focus changes in organization and evaluation to improve the portfolio as a dynamic process.

(Rev Med Chile 2011; 139: 45-53).

Key words: Portfolios; Psychometrics; Students, Medical; Teaching.

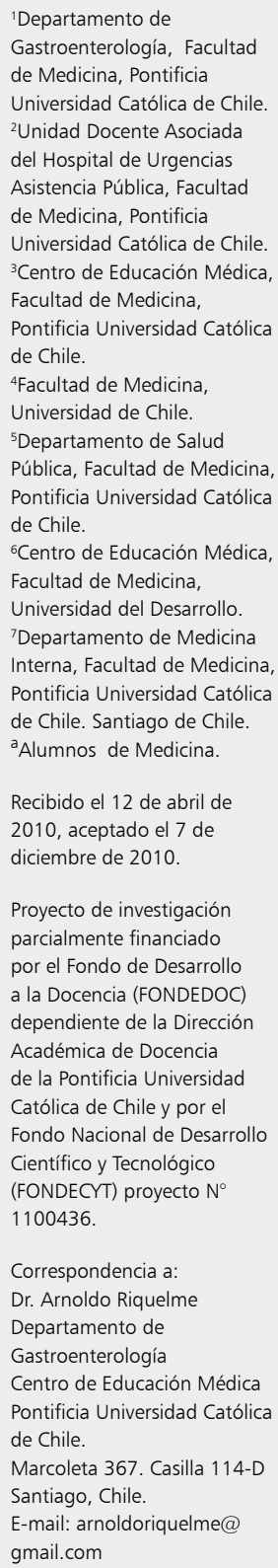

$\mathrm{D}$ esde 1993, la Escuela de Medicina de la Pontificia Universidad Católica de Chile (EMPUC) lleva realizando de manera exitosa una reforma curricular ${ }^{1,2}$, incluyendo modificaciones en el curso integrado de clínica de cuarto año, donde los estudiantes adquieren conocimientos y destrezas clínicas, mediante actividades teóricas y prácticas en contacto con pacientes. El año 2007 se introdujo el Portafolio como metodología de aprendizaje y de evaluación de lo que los alumnos "hacen" durante su práctica en las rotaciones clínicas, para contrarrestar el énfasis en la memorización y sobrecarga de información percibidos por los alumnos en la encuesta DREEM de ambiente educacional ${ }^{3,4}$. 
Un portafolio es una colección del trabajo del estudiante, fruto de un proceso de aprendizaje independiente, que provee de evidencia de los logros en relación a la aplicación del conocimiento, desarrollo de destrezas clínicas con pacientes en un período de tiempo ${ }^{5}$. De esta manera, el portafolio incorpora la reflexión al proceso de revisión y análisis de una experiencia clínica promoviendo el pensamiento crítico ${ }^{6}$.

El portafolio puede incluir consultas video registradas, evaluaciones entre pares, revisiones de la literatura, proyectos de implementación de calidad, y cualquier otro material que demuestre aprendizaje y reflexión en el estudiante ${ }^{7}$. El portafolio de pregrado incluye el análisis de casos clínicos (2007) y 4 casos con integración vertical y horizontal de conceptos fisiopatológicos, epidemiológicos y bioéticos. La eficacia de un portafolio depende de una estricta supervisión por parte de un docente experto, tiempo protegido y una buena organización $^{6,8-11}$. Por esta razón es que parte del proceso de implementación del portafolio incluyó el desarrollo y validación de una encuesta que fue aplicada a fin de los años académicos 2007 y 2008 como herramienta de evaluación de la introducción de esta nueva metodología de innovación en docencia.

Los objetivos de este estudio son describir el proceso de desarrollo de la encuesta de evaluación de un portafolio genérico de pregrado y su posterior validación incluyendo análisis de propiedades psicométricas del instrumento y de los resultados obtenidos en los años 2007 y 2008.

\section{Métodos}

El diseño e implementación del portafolio en docencia de pregrado fue llevado a cabo durante el año 2007, como parte de la monitorización de este instrumento de innovación en docencia, al término de los años 2007 y 2008. Se utilizó la metodología de la teoría fundada mediante técnica de grupo focal por alumnos y docentes ${ }^{12}$. Se identificaron los aspectos relacionados con el aprendizaje, enseñanza y evaluación del portafolio en pregrado, incluyendo aspectos genéricos del ambiente educacional ${ }^{12-14}$. Posteriormente un grupo de docentes clínicos con experiencia en docencia tutorial depuraron y priorizaron la información a través de 3 rondas para determinar los ítems definitivos de la encuesta ${ }^{15}$.
Para la cuantificación de la percepción o grado de acuerdo de los encuestados con las afirmaciones enunciadas en cada ítem, se utilizó una escala de Likert de 0 a 4 , desde: $0=$ Completamente en Desacuerdo (CD); $1=$ en Desacuerdo (D); $2=$ No está Seguro o no tiene ninguna opinión (NS); $3=\mathrm{de}$ Acuerdo (A); hasta $4=$ Completamente de Acuerdo (CA) y los ítems con enunciados negativos se registraron con puntaje inverso.

Para evaluar la validez del constructo de las subescalas se usó un análisis factorial exploratorio seguido de una rotación Varimax ${ }^{16}$. Los factores fueron elegidos usando una combinación de los siguientes 3 criterios: 1) Criterio Kaiser-Guttman, en el que se incluyeron todos los factores con Eigenvalue $>11^{17,18} ; 2$ ) Criterio de Cattell, mediante la identificación del punto de inflexión de la curva del gráfico de sedimentación ${ }^{19}$; y 3 ) Los factores identificados en el análisis factorial exploratorio deben incluir la mayoría de la varianza (> 50\% del total).

La confiabilidad de la encuesta fue evaluada por método de Cronbach y la consistencia interna del instrumento fue expresada como coeficiente alfa de Cronbach en escala de 0 a $1^{20}$.

Los ítems, puntajes por dominio y puntajes globales de la encuesta fueron expresados en promedios y desviación estándar y las comparaciones entre los puntajes obtenidos el 2007 y 2008 fueron comparados mediante test de $t$ de Student. Los valores $p<0,05$ fueron considerados estadísticamente significativos. Los análisis estadísticos incluyendo la evaluación de confiabilidad mediante el cálculo del coeficiente alfa de Cronbach fueron realizados mediante programa SPSS, Statistics 17 para Windows. Versión 17. 0. 0, del 23 de agosto de 2008 (SPSS, Inc., Chicago, IL).

\section{Resultados}

La validez de contenido fue llevado a cabo por el panel Delfi conformado por 12 tutores clínicos y un educador médico, identificando en la primera ronda 61 aspectos relacionados con el portafolio en docencia de pregrado para luego priorizarlos en 43 ítems que conformaron la encuesta definitiva en la tercera ronda asegurando una adecuado muestreo de cada una de las sub-escalas (Tabla 1 ). Los ítems 2, 9, 11, 13, 17, 29, 33, 34, 35 y 42 son enunciados negativos, por lo que en la tabulación de datos se registraron con puntaje inverso. El 
puntaje total es de 172 puntos para los 43 ítems. La validez de constructo fue examinada usando el análisis factorial exploratorio seguido de la rotación Varimax de los datos, resultando en 13 factores con Eigenvalue $>1$. Los 13 factores juntos daban cuenta del 72,2\% de la varianza. El punto de inflexión de las curva del gráfico de sedimentación (Figura 1) se observó entre los factores 6 y 7 . Luego, forzamos el análisis factorial a 6 factores, cuya distribución se ilustra en el gráfico de sedimentación (Figura 1), dando cuenta a su vez del 51,8\% de la varianza. La distribución de los ítems fue: Factor 1: ítems 1, 3, 4, 5, 8, 14, 15, 16, 18, 19, 20, 21, 22, $23,25,27,28,30,32,37,38,39,40,41,42$, у 43; Factor 2: ítems 2, 11, 17 y 31; Factor 3: ítems 7, 35 y 36; Factor 4: ítems 6, 9, 10 y 29; Factor 5: ítem 33; y Factor 6: ítems 12, 13, 24, 26 y 34. Los 6 factores o sub-escalas definitivas fueron nominadas por el equipo de investigación en base al contenido de los enunciados de los ítems contenidos en cada factor: Factor 1: "Aprendizaje del Estudiante" (21 ítems con un puntaje máximo de 104 puntos), Factor 2: "Organización y Evaluación" (4 ítems con un puntaje máximo de 16 puntos), Factor 3: "Metodología Docente" (3 ítems con un puntaje máximo de 12 puntos), Factor 4: "Apoyo Docente" (4 ítems con un puntaje máximo de 16 puntos), Factor 5: "Creatividad" (un ítem con un puntaje máximo de 4 puntos) y finalmente, el Factor 6:
"Integración" (contiene 5 ítems con un puntaje máximo de 20 puntos). Los ítems contenidos en cada dominio se muestran en la Tabla 1.

Los resultados de la encuesta fueron confiables según el análisis de consistencia interna, con un coeficiente de Cronbach alfa de 0,9 para el puntaje global de la encuesta.

La encuesta fue aplicada en los años 2007 y 2008 en los alumnos de $4^{\circ}$ año, en relación al curso integrado de clínica. En el año 2007 fue aplicada a 97 alumnos, con un porcentaje de respuesta total de 99,9\%. En tanto que el año 2008 fueron 100 los alumnos encuestados, siendo $99,2 \%$ su porcentaje de respuesta. El puntaje global promedio obtenido en cada año fue de 106,2 $\pm 21,2(61,7 \%)$ y $104,6 \pm$ $34(60,8 \%)$ para el 2007 y 2008, respectivamente $(\mathrm{p}=\mathrm{NS})$. Los dominios mejor evaluados por los estudiantes fueron la "Metodología Docente" con 72,7\% (2007) y la "Creatividad" con 68,8\% (2007) del máximo puntaje esperado para cada dominio, sin diferencias significativas con el año 2008. Por otro lado, el dominio peor evaluado por los estudiantes fue la "Organización y Evaluación" con sólo 45,4\% (2007). No se observaron diferencias significativas en el análisis según dominios o subescalas al comparar los promedios del 2007 y 2008. Los ítems 7, 8, 10, 12, 16, 27, 29, 31 y 32 presentaron diferencias significativas en sus respuestas entre los años 2007 y 2008 (Tabla 1).

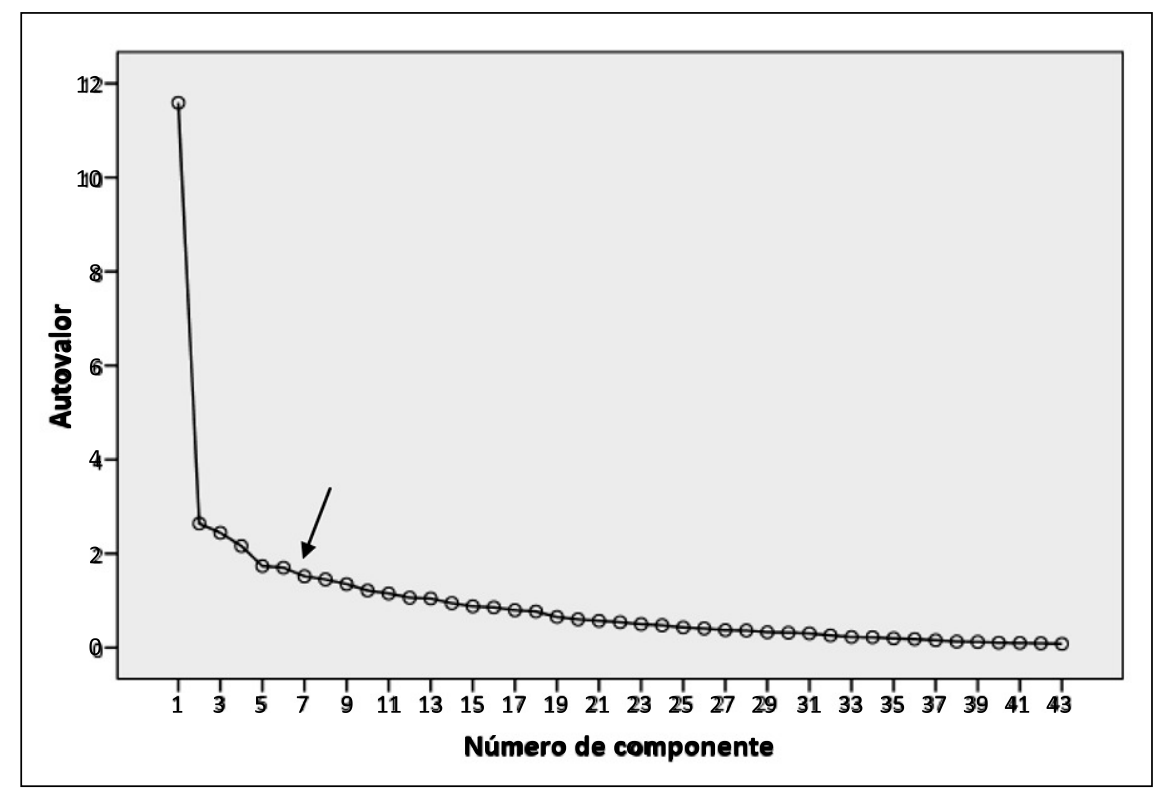

Figura 1. Gráfico de sedimentación de los factores o dominios identificados en el análisis factorial de la encuesta de evaluación del Portafolio de 43 ítems. La flecha indica el punto de inflexión de la curva entre los factores 6 y 7. 
Tabla 1. Resultado de la encuesta de la percepción de los estudiantes del portafolio de pregrado en medicina

\begin{tabular}{|c|c|c|c|c|c|c|c|}
\hline & & 2007 & & & 2008 & & \\
\hline & $\begin{array}{l}\text { Porcentaje } \\
\text { de } \\
\text { respuesta }\end{array}$ & Promedio & $\begin{array}{l}\text { Desviación } \\
\text { estándar }\end{array}$ & $\begin{array}{l}\text { Porcentaje } \\
\text { de } \\
\text { respuesta }\end{array}$ & Promedio & $\begin{array}{l}\text { Desviación } \\
\text { estándar }\end{array}$ & $\begin{array}{c}\text { Valor } \\
\mathbf{p}\end{array}$ \\
\hline Aprendizaje del estudiante & & & & & & & \\
\hline $\begin{array}{l}\text { 01. El Portafolio fue de utilidad en } \\
\text { mi aprendizaje clínico aplicado }\end{array}$ & 100 & 3,03 & 0,99 & 99 & 2,92 & 1,16 & 0,470 \\
\hline $\begin{array}{l}\text { 18. El portafolio potenció mis forta- } \\
\text { lezas clínicas }\end{array}$ & 100 & 2,66 & 1 & 100 & 2,48 & 1,12 & 0,237 \\
\hline $\begin{array}{l}\text { 21. Siento que el portafolio me está } \\
\text { preparando bien para mi profesión }\end{array}$ & 100 & 2,27 & 1,1 & 99 & 2,15 & 1,23 & 0,487 \\
\hline $\begin{array}{l}\text { 20. El Portafolio me ayudó en el } \\
\text { aprendizaje independiente }\end{array}$ & 100 & 2,98 & 0,97 & 99 & 2,88 & 1,13 & 0,504 \\
\hline $\begin{array}{l}\text { 04. El portafolio es un buen comple- } \\
\text { mento a los métodos de evaluación } \\
\text { pre-existentes }\end{array}$ & 100 & 2,55 & 1,12 & 100 & 2,62 & 1,22 & 0,66 \\
\hline $\begin{array}{l}\text { 19. El Portafolio me ayudó a re- } \\
\text { conocer y mejorar mis debilidades } \\
\text { relacionadas con mi práctica clínica }\end{array}$ & 100 & 2,49 & 1,17 & 99 & 2,39 & 1,21 & 0,594 \\
\hline $\begin{array}{l}05 . \text { El portafolio me ayuda a inte- } \\
\text { grar conocimientos teóricos y des- } \\
\text { trezas clínicas }\end{array}$ & 100 & 2,97 & 0,9 & 99 & 2,81 & 1,16 & 0,278 \\
\hline $\begin{array}{l}\text { 27. El portafolio es un complemento } \\
\text { a las rotaciones clínicas }\end{array}$ & 100 & 2,47 & 1,18 & 99 & 2,51 & 1,35 & 0,005 \\
\hline $\begin{array}{l}\text { 42. El portafolio debería eliminarse } \\
\text { el próximo año }\end{array}$ & 100 & 2,64 & 1.16 & 99 & 2,46 & 1,42 & 0,321 \\
\hline $\begin{array}{l}\text { 28. El portafolio ofrece una posibi- } \\
\text { lidad de reflexionar en la práctica } \\
\text { clínica }\end{array}$ & 100 & 2,61 & 0,99 & 99 & 2,69 & 1,15 & 0,865 \\
\hline $\begin{array}{l}\text { 40. El portafolio me ha ayudado } \\
\text { a utilizar la información en forma } \\
\text { racional y aplicada al paciente }\end{array}$ & 100 & 2,93 & 0,81 & 99 & 2,73 & 1,16 & 0,162 \\
\hline $\begin{array}{l}\text { 25. El Portafolio es un método que } \\
\text { me ayudará en el internado y en mi } \\
\text { futuro como profesional }\end{array}$ & 100 & 2,39 & 1,11 & 99 & 2,19 & 1,3 & 0,248 \\
\hline $\begin{array}{l}\text { 30. El Portafolio se enfoca en con- } \\
\text { tenidos relevantes para la práctica } \\
\text { profesional }\end{array}$ & 100 & 2,77 & 0,82 & 99 & 2,53 & 1,16 & 0,086 \\
\hline $\begin{array}{l}\text { 39. El portafolio ha sido una herra- } \\
\text { mienta de gran utilidad en la estruc- } \\
\text { turación del diagnóstico clínico }\end{array}$ & 100 & 2,64 & 0,97 & 99 & 2,46 & 1,19 & 0,236 \\
\hline 15. Disfruté haciendo mi portafolio & 98,97 & 1,46 & 1,16 & 100 & 1,54 & 1,31 & 0,646 \\
\hline $\begin{array}{l}\text { 37. El portafolio estimula la origi- } \\
\text { nalidad }\end{array}$ & 100 & 1,94 & 1,21 & 99 & 2,19 & 1,41 & 0,177 \\
\hline $\begin{array}{l}\text { 32. El portafolio estimula la resolu- } \\
\text { ción de problemas usando el razo- } \\
\text { namiento clínico }\end{array}$ & 100 & 3,1 & 0,64 & 99 & 2,84 & 1,12 & 0,044 \\
\hline
\end{tabular}


Tabla 1. Resultado de la encuesta de la percepción de los estudiantes del portafolio de pregrado en medicina (continuación)

\begin{tabular}{|c|c|c|c|c|c|c|c|}
\hline & & 2007 & & & 2008 & & \\
\hline & $\begin{array}{l}\text { Porcentaje } \\
\text { de } \\
\text { respuesta }\end{array}$ & Promedio & $\begin{array}{l}\text { Desviación } \\
\text { estándar }\end{array}$ & $\begin{array}{l}\text { Porcentaje } \\
\text { de } \\
\text { respuesta }\end{array}$ & Promedio & $\begin{array}{c}\text { Desviación } \\
\text { estándar }\end{array}$ & $\begin{array}{c}\text { Valor } \\
\text { p }\end{array}$ \\
\hline $\begin{array}{l}\text { 14. El portafolio fue un reflejo de } \\
\text { mi evolución durante el año }\end{array}$ & 100 & 2,28 & 1,26 & 100 & 2,06 & 1,23 & 0,219 \\
\hline $\begin{array}{l}\text { 22. El Portafolio pone énfasis en } \\
\text { el conocimiento aplicado al pro- } \\
\text { blema de mi paciente }\end{array}$ & 100 & 3 & 0,84 & 99 & 2,85 & 1,14 & 0,291 \\
\hline $\begin{array}{l}\text { 41. El portafolio refuerza mis } \\
\text { habilidades relacionadas con la } \\
\text { anamnesis y el examen físico }\end{array}$ & 100 & 2,44 & 1,11 & 99 & 2,59 & 1,16 & 0,38 \\
\hline $\begin{array}{l}\text { 38. El portafolio promueve la res- } \\
\text { ponsabilidad }\end{array}$ & 100 & 2,98 & 0,89 & 99 & 2,9 & 1,09 & 0,573 \\
\hline $\begin{array}{l}\text { 43. Estoy orgulloso del producto } \\
\text { final obtenido }\end{array}$ & 100 & 2,91 & 1,01 & 99 & 2,66 & 1,24 & 0,123 \\
\hline $\begin{array}{l}\text { 16. Las pautas de evaluación en- } \\
\text { tregadas fueron de utilidad para } \\
\text { el desarrollo de los casos clínicos }\end{array}$ & 100 & 2,08 & 1,23 & 98 & 2,61 & 1,27 & 0,003 \\
\hline $\begin{array}{l}\text { 03. Recibí un feedback formativo } \\
\text { adecuado durante el proceso de } \\
\text { elaboración del portafolio }\end{array}$ & 100 & 2,72 & 1,34 & 100 & 2,83 & 1,33 & 0,569 \\
\hline $\begin{array}{l}\text { 08. El portafolio estuvo bien or- } \\
\text { ganizado }\end{array}$ & 100 & 0,99 & 1,06 & 100 & 2,51 & 1,25 & 0,0001 \\
\hline $\begin{array}{l}\text { 23. El portafolio es un método } \\
\text { justo de evaluación }\end{array}$ & 100 & 2,35 & 1,1 & 99 & 2,35 & 1,3 & 0,339 \\
\hline $\begin{array}{l}\text { Promedio del Dominio } 1 \text { (puntaje } \\
\text { máximo }=104 \text { ) }\end{array}$ & 98,97 & 65,64 & 12,85 & 98 & 65,72 & 8,24 & 0,979 \\
\hline Organización y Evaluación & & & & & & & \\
\hline $\begin{array}{l}\text { 02. El tiempo requerido fue ex- } \\
\text { cesivo }\end{array}$ & 100 & 0,81 & 1,02 & 100 & 0,84 & 1,04 & 0,862 \\
\hline $\begin{array}{l}\text { 11. No tuve tiempo protegido } \\
\text { para hacer el trabajo relacionado } \\
\text { con el portafolio }\end{array}$ & 100 & 1,57 & 1,19 & 99 & 1,58 & 1,29 & 0,961 \\
\hline $\begin{array}{l}\text { 17. Creo que la copia en el porta- } \\
\text { folio constituyó un problema entre } \\
\text { mis compañeros }\end{array}$ & 100 & 3,18 & 0,96 & 99 & 2,98 & 1,05 & 0,175 \\
\hline 31. El portafolio tiene reglas claras & 100 & 1,71 & 1,44 & 99 & 2,71 & 1,21 & 0,0001 \\
\hline $\begin{array}{l}\text { Promedio del Dominio } 2 \text { (puntaje } \\
\text { máximo=16) }\end{array}$ & 100 & 7,27 & 3,95 & 99 & 8,1 & 3,99 & 0,776 \\
\hline Metodología docente & & & & & & & \\
\hline $\begin{array}{l}\text { 07. Los profesores involucrados en } \\
\text { el portafolio son motivados }\end{array}$ & 100 & 3,29 & 1,03 & 100 & 2,73 & 1,21 & 0,001 \\
\hline $\begin{array}{l}\text { 36. Los análisis son centrados en } \\
\text { el paciente }\end{array}$ & 98,97 & 3,13 & 0,76 & 99 & 2,89 & 0,93 & 0,053 \\
\hline
\end{tabular}


Tabla 1. Resultado de la encuesta de la percepción de los estudiantes del portafolio de pregrado en medicina (continuación)

\begin{tabular}{|c|c|c|c|c|c|c|c|}
\hline & \multicolumn{3}{|c|}{2007} & \multicolumn{3}{|c|}{2008} & \multirow[b]{2}{*}{$\begin{array}{c}\text { Valor } \\
\mathbf{p}\end{array}$} \\
\hline & $\begin{array}{l}\text { Porcentaje } \\
\text { de } \\
\text { respuesta }\end{array}$ & Promedio & $\begin{array}{l}\text { Desviación } \\
\text { estándar }\end{array}$ & $\begin{array}{l}\text { Porcentaje } \\
\text { de } \\
\text { respuesta }\end{array}$ & Promedio & $\begin{array}{l}\text { Desviación } \\
\text { estándar }\end{array}$ & \\
\hline $\begin{array}{l}\text { 35. El apoyo material y tecnológico } \\
\text { no es el adecuado }\end{array}$ & 100 & 2,31 & 1,08 & 98 & 2,55 & 1,1 & 0,124 \\
\hline $\begin{array}{l}\text { Promedio del Dominio } 3 \text { (puntaje } \\
\text { máximo }=12 \text { ) }\end{array}$ & 98,97 & 8,72 & 1,57 & 98 & 8,17 & 0,51 & 0,593 \\
\hline \multicolumn{8}{|l|}{ Apoyo docente } \\
\hline $\begin{array}{l}\text { 09. No tuve oportunidad de mejorar } \\
\text { aquellos aspectos evaluados como } \\
\text { deficientes en el feedback }\end{array}$ & 100 & 2,77 & 1,03 & 99 & 2,79 & 1,19 & 0,926 \\
\hline $\begin{array}{l}\text { 10. Las actividades del portafolio } \\
\text { fueron bien coordinadas con el resto } \\
\text { de las actividades del año. }\end{array}$ & 98,97 & 1,18 & 1,07 & 99 & 1,76 & 1,21 & 0 \\
\hline $\begin{array}{l}\text { 06.- Los profesores tienen paciencia } \\
\text { con los alumnos que presentan } \\
\text { dificultades }\end{array}$ & 100 & 2,83 & 0,9 & 99 & 2,83 & 1,04 & 0,98 \\
\hline $\begin{array}{l}\text { 29. El portafolio es un método de } \\
\text { aprendizaje pasivo. }\end{array}$ & 100 & 2,68 & 1,11 & 99 & 2,24 & 1,25 & 0,011 \\
\hline $\begin{array}{l}\text { Promedio del Dominio } 4 \text { (puntaje } \\
\text { máximo }=16 \text { ) }\end{array}$ & 98,97 & 9,46 & 3,17 & 98,97 & 9,62 & 2,03 & 0,935 \\
\hline \multicolumn{8}{|l|}{ Creatividad } \\
\hline $\begin{array}{l}\text { 33. El portafolio es una transcripción } \\
\text { de los mismos contenidos de las } \\
\text { clases teóricas }\end{array}$ & 100 & 2,75 & 1,06 & 99 & 2,55 & 1,18 & 0,198 \\
\hline $\begin{array}{l}\text { Promedio del Dominio } 5 \text { (puntaje } \\
\text { máximo }=4 \text { ) }\end{array}$ & 100 & 2,75 & 1,06 & 99 & 2,55 & 1,18 & 0,198 \\
\hline \multicolumn{8}{|l|}{ Integración } \\
\hline $\begin{array}{l}\text { 24. El portafolio me permitió aplicar } \\
\text { conocimientos básicos aprendidos } \\
\text { en años previos }\end{array}$ & 100 & 2,58 & 0,97 & 99 & 2,18 & 1,35 & 0,173 \\
\hline $\begin{array}{l}\text { 26. El portafolio contempla aspectos } \\
\text { éticos aplicados a los problemas de } \\
\text { mis pacientes }\end{array}$ & 100 & 2,43 & 1,03 & 99 & 2,36 & 1,26 & 0,673 \\
\hline $\begin{array}{l}\text { 34. Las variables epidemiológicas y } \\
\text { el entorno familiar y social del pa- } \\
\text { ciente no son considerados }\end{array}$ & 98,97 & 2,71 & 0,96 & 99 & 2,87 & 0,97 & 0,247 \\
\hline $\begin{array}{l}\text { 12. El número de casos incluidos al } \\
\text { final fue adecuado }\end{array}$ & 100 & 3,19 & 0,89 & 100 & 2,71 & 1,26 & 0,003 \\
\hline $\begin{array}{l}\text { 13. Los análisis adicionales me pare- } \\
\text { cen omitibles e irrelevantes respecto } \\
\text { a los casos clínicos básicos }\end{array}$ & 100 & 1,5 & 1,28 & 99 & 1,25 & 1,25 & 0,182 \\
\hline $\begin{array}{l}\text { Promedio del Dominio } 6 \text { (puntaje } \\
\text { máximo }=20 \text { ) }\end{array}$ & 98,97 & 12,4 & 3,1 & 99 & 11,38 & 3,17 & 0,62 \\
\hline $\begin{array}{l}\text { Promedio Global (puntaje máxi- } \\
\text { mo=172) }\end{array}$ & 99,9 & 106,15 & 21,21 & 99,16 & 104,63 & 33,95 & 0,909 \\
\hline
\end{tabular}


En el primer dominio ("Aprendizaje del Estudiante") el puntaje observado fue de $65,4 \pm$ 12,9 (62,9\% de puntaje máximo) el año 2007, sin diferencias significativas con respecto al año 2008. El ítem 32 (El portafolio estimula la resolución de problemas usando el razonamiento clínico; $3,1 \pm 0,64)$ presentó el mayor puntaje en el año 2007, mientras que el ítem 8 (El portafolio estuvo bien organizado; $0,98 \pm 1,06$ ) presentó el puntaje más bajo del 2007, experimentando un aumento significativo el $2008(2,51 \pm 1,25 ; \mathrm{p}=0,0001)$, probablemente debido a los cambios focalizados implementados durante el 2008, destinados a mejorar aspectos organizacionales. En el segundo dominio ("Organización y Evaluación") se observó un promedio de 7,27 \pm 3,95 el año 2007 (45,4\% del puntaje máximo), que experimentó un ascenso por sobre el 50\% $(8,1 \pm 3,99 ; 50,6 \%$ del puntaje máximo) durante el año 2008 con un aumento significativo en el ítem 31 (El portafolio tiene reglas claras) con 1,71 $\pm 1,44$ (2007) a 2,71 $\pm 1,21$ (2008) con $p=0,0001$. Por otro lado, pese a la reducción de los casos clínicos solicitados el 2008, persiste la sensación que el trabajo requerido es excesivo $0,81 \pm 1,02$ y $0,84 \pm 1,04(p=0,862)$, ligado a la percepción de la falta de tiempo protegido para la realización del portafolio (ítem 11) con $1,57 \pm 1,19$ y $1,58 \pm 1,29$ los años 2007 y 2008 $(\mathrm{p}=0,961)$. En el tercer dominio ("Metodología Docente") el promedio fue de 8,72 \pm 1,57 el 2007, que corresponde al 72,7\% del puntaje máximo, correspondiendo a uno de los dominios con percepción positiva por parte de los estudiantes. En relación al cuarto dominio ("Apoyo Docente") el promedio fue de 9,46 \pm 3 ,17 el 2007, que corresponde al 59,1\% del puntaje máximo. Destaca un aumento significativo en el ítem 10 (Las actividades del portafolio fueron bien coordinadas con el resto de las actividades del año) de 1,18 \pm 1,29 el 2007 a $1,76 \pm 1,21$ el $2008(\mathrm{p}=0,0001)$. El quinto dominio ("Creatividad") consta de un ítem que mantuvo promedios muy positivos sin diferencias significativas al comparar los años 2007 y $2008(2,75 \pm 1,06 ; 68,8 \%$ del máximo puntaje, respecto de $2,55 \pm 1,18$; que corresponde al $63,8 \%$ del máximo puntaje, con $\mathrm{p}=0,298$ ). Finalmente, en el sexto dominio ("Integración") presenta un promedio de 12,4 $\pm 3,1$ el 2007, que corresponde al $62 \%$ del puntaje máximo para el dominio, destacando que pese a que el número de casos clínicos es considerado como adecuado, se observa una reducción significativa en el ítem 12 de 3,19 $\pm 0,89$ el 2007 a $2,71 \pm 1,26$ el $2008(p=0,003)$.

\section{Discusión}

El portafolio ofrece al estudiante evidencias de su aprendizaje, logros, y desarrollo de sus competencias ${ }^{21}$. En muchas universidades norte americanas y europeas existe un proceso de implementación de portafolio a lo largo de toda la carrera de medicina, siendo considerado una opción válida a los métodos de evaluación tradicionales ${ }^{22-24}$. Los mismos estudiantes consideran el portafolio útil para explorar el arte de la profesión y no sólo para adquirir competencias técnicas ${ }^{25}$. Es así como el portafolio se considera un complemento válido a los exámenes escritos (conocimiento) y exámenes clínicos estructurados (ECOE) que miden destrezas clínicas ${ }^{7,25}$. Sin embargo, son muchas las controversias en relación al portafolio y su introducción en el curriculum. Entre los aspectos más críticos se identifican el contar con el tiempo necesario para su adecuado desarrollo, lo que constituye uno de los aspectos evaluados negativamente en nuestra encuesta ${ }^{8-10}$. La validez del instrumento representa un punto crítico según algunos autores, debido a que los portafolios generalmente contienen evidencia cualitativa más que cuantitativa, y además los docentes expresan juicios cualitativos sobre el proceso del estudiante $\mathrm{e}^{10,26}$.

El diseño y la implementación del portafolio en la EMPUC, consideró el desarrollo y validación de una encuesta de evaluación para introducirlo de manera rigurosa y fundamentar los cambios ocurridos ${ }^{27}$.

El proceso de implementación tuvo que lidiar con dificultades operacionales que están bien descritas en la literatura y principalmente con el cambio cultural relacionado a toda nueva metodología, con resistencia por parte de los alumnos, debido a la ansiedad ante lo desconocido y el tiempo necesario para la escritura del portafolio ${ }^{6}$. El año 2007 se comenzó con el proceso de diseño e implementación del portafolio y se incluyó la construcción y validación de la encuesta de evaluación de las percepciones del portafolio por parte de los alumnos como una forma de monitorización cuantitativa el proceso. El instrumento cuenta con validez de contenido ya que incorpora todos 
los aspectos identificados por los grupos focales. Desde el punto de vista del muestreo, la encuesta cuenta con una cantidad adecuada de ítems con lo cual se asegura la medición de los aspectos relevantes para luego ser agrupados en dominios que surgen de un análisis factorial que aseguran la validez de constructo. Respecto a la confiabilidad de las encuestas, se considera como excelente un valor $>0,8$ y nuestra encuesta obtuvo un valor alfa de $0,9^{20,28-30}$.

Respecto a la validez de contenido, en el pregrado, el portafolio debe incluir el análisis de casos clínicos obtenidos de sus rotaciones clínicas hospitalarias y/o ambulatorias, ofreciendo una retroalimentación frecuente por parte del docente, con énfasis en el análisis crítico de dichos casos y su adecuada integración con ramos de ciencias básicas, fisiopatología, bioética y el internado ${ }^{24,29,30}$. Si se utiliza el portafolio en un contexto de elevada complejidad y para evaluar altos niveles de competencia, como por ejemplo en posgrado, sería necesario considerar otros contenidos como registro de procedimientos y análisis de eventos críticos ${ }^{27,31}$.

Los resultados del 2007 según ítem, dominio y puntaje global son muy positivos y respaldan la percepción de utilidad y complementariedad del portafolio con el resto de las instancias de aprendizaje y evaluación del curso integrado de cuarto año. Los dominios mejor evaluados por los estudiantes fueron la "Metodología Docente", reflejando un buen grupo de docentes motivados, guiando el proceso de aprendizaje de los alumnos y la "Creatividad", que se podría explicar por la oportunidad de desarrollar un producto original y personal que demuestre lo aprendido en la práctica clínica, integrando conocimientos de otros cursos de forma atractiva para el alumno. Por otro lado, el dominio peor evaluado por los estudiantes fue la "Organización y Evaluación”. Debemos destacar que tanto el dominio "Organización y Evaluación" como la mayoría de los ítems deficientes del año 2007, corresponden a aspectos organizacionales y de reglamentación que motivaron profundos cambios en el proceso 2008, con capacitación docente, reducción del número de casos clínicos, reglamentación explícita dentro de la guía de estudio a los estudiantes, entrega de las pautas de evaluación y adquisición de cámaras digitales para cada una de las unidades docentes donde se desarrollaba la actividad clínica. Estos cambios, fueron evaluados positivamente por parte de los estudiantes con una mejoría significativa de los ítems relacionados con organización, evaluación y reglamentación al repetir la encuesta el año $2008^{6-8}$. En el año 2009 se implementó un nuevo sistema de revisión con evaluaciones formativas frecuentes realizadas por ayudantes-alumnos de quinto año que se consolidó durante el año 2010. Desconocemos el impacto de dicho cambio en la percepción de los estudiantes y por esta razón decidimos excluir dichos años del análisis.

En conclusión, la encuesta de evaluación de la percepción de los estudiantes del portafolio de pregrado en Medicina es válida y confiable. La encuesta confirmó una percepción positiva de los estudiantes de las fortalezas del portafolio, tales como el estímulo a la creatividad y el profesionalismo de los alumnos. Las debilidades identificadas del proceso de implementación permitió la focalización de esfuerzos en cambios organizacionales y de evaluación con la finalidad de mejorar el portafolio en proceso evolutivo y dinámico en el tiempo.

\section{Referencias}

1. Moreno R, Velasco N. [Curricular changes in the School of Medicine of the Pontificia Universidad Católica de Chile]. Rev Chil Cir 1994; 46: 3336.

2. Rosso P, Velasco N, Moreno R. [Undergraduate curriculum reform at the Pontifical Catholic University Medical School: aims, methodology and advance status]. Rev Med Chile 1997; 125: 796-807.

3. Roff S, Mcaleer S, Harden RM, Al-Qahtani M, Uddin AA, Groenen G, et al. Development and Validation of the Dundee Ready Education Environment Measure (DREEM). Medical Teacher 1997; 19: 295-9.

4. Davis MH, Friedman BD, Harden RM, Howie P, Ker J, Mcghee C, et al. Portfolio assessment in medical students' final examinations. Medical Teacher 2001; 23: 357-66.

5. Davis MH, Ponnanperuma GG. Portfolios, projects and dissertations. En: Dent JA, Harden RM, ed. A practical guide for medical teachers (Second Edition). London: Elsevier Churchill Livingstone publishers, 2005; 346-56.

6. Buckley S, Coleman J, Davison I, Khan Ks, Zamora J, Malick S, et al. The educational effects of portfolios on undergraduate student learning: A Best Evidence Medical Education (BEME) Systematic Review. BEME Guide no. 11. Medical Teacher 2009; 31: 282-98.

7. Epstein R. Assessment in Medical Education. N Engl J Med 2007; 356: 387-96. 
8. Driessen E, Van Tartwijk J, Van Der Vleuten C, Wass V. Portfolios in medical education: why do they meet with mixed success? A systematic review. Med Educ 2007; 41: 1224-33.

9. Driessen E. Are learning portfolios worth the effort? Yes. BMJ 2008; 337: a513.

10. Norman G. Are learning portfolios worth the effort? No. BMJ 2008; 337: a513-a514.

11. Miller GE. The assessment of Clinical Skills/Competence/Performance. Acad Med 1990; 65: s63-7.

12. Reed B. Organizational role analysis. In: Cooper C, ed. Developing Social Skills in Managers. Advances in Group Training. London: Mcmillan, 1976; 89-102.

13. Riquelme A, Fuentes G, Jeria A, Méndez I, Aranís C, Larios G, et al. Ambiente educacional y calidad de la docencia en la Escuela de Medicina. Ars Medica 2008; 15, 15: 125-39.

14. Riquelme A, Herrera C, Aranis C, Oporto J, Padilla O. Psychometric analyses and internal consistency of PHEEM questionnaire to measure the clinical learning environment in the clerkship of a Medical School in Chile. Medical Teacher 2009; 31(6): e221-5.

15. Hasson F, Keeney S, Mckenna H. Research guidelines for the Delphi survey technique. J Adv Nurs 2000; 32(4): 1008-15.

16. Boor K, Scheele F, Van Der Vleuten Cpm, Scherpbier AJJA, Teunissen PW, Sijtsma K. Psychometric properties of an instrument to measure the clinical learning environment. Med Educ 2007; 41: 92-9.

17. Field A. Discovering statistics using SPSS for Windows. London: Sage Publications Limited, 2000.

18. Field A. Discovering statistics using SPSS for Windows (Second Edition). London: Sage Publications Limited, 2005.

19. Cattell RB. The scree test for a number of factors. Multivariate Behavioural Research 1966; 1: 245-76.

20. Cronbach LJ. Coefficient alpha and the internal structure of tests. Psychometrika 1951; 16: 297-34.

21. Driessen E, Overeem K, Van Tartwijk J, Van Der Vleuten C, Muijtjens A. Validity of portfolio assessment: which qualities determine ratings? Med Educ 2006; 40: 862-6.

22. Davis M, Ponnamperuma G, Ker J. Students' perceptions of a portfolio assessment process. Med Educ 2009; 43: 89-98.

23. Robinson MA. College Students' attitudes toward portfolio assessment as an alternative to traditional tests. It Forum 2000. Disponible en: htpp://it.coe.uga.edu/itforum/paper47/paper47.htm [consultado el 12 de junio de 09].

24. Dannefer EF, Henson LC. The portfolio approach to competency-based assessment at the Cleveland Clinic Lerner College of Medicine. Acad Med 2007; 82: 493 502.

25. Nairn S, O'brien E, Traynor V, Williams G, Chapple M, Johnson S. Student nurses' knowledge, skills and attitudes towards the use of portfolios in a school of nursing. J Clin Nurs 2006; 15: 1509-20.

26. Webb C, Endacott R, Gray M, Jasper M, Mcmullan M, Scholes J. Evaluating portfolio assessment systems: what are the appropriate criteria? Nurse Educ Today 2003; 23: 600-9.

27. Meeus W, Van Petegem P, Engels N. Validity and reliability of portfolio assessment in pre-service teacher education. Assessment \& Evaluation in Higher Education 2009; 34: 401-13.

28. Streiner D, Norman G. Health Measurement Scale: A Practical Guide to their Development and Use (Fourth Edition). Oxford: Oxford University Press, 2008; 77102.

29. Driessen E, Van Der Vleuten C, Schuwirth L, Van Tartwijk J, Vermunt J. The use of qualitative research criteria for portfolio assessment as an alternative to reliability evaluation: a case study. Med Educ 2005; 39: 214-20.

30. Pitts J, Coles C, Thomas P. Enhancing reliability in portfolio assessment: "shaping" the portfolio. Medical Teacher 2001; 23: 351-6.

31. Gadbury-Amyot C, Kim J, Palm R, Mills E, Noble E, Overman P. Validity and reliability of portfolio assessment of competency in a baccalaureate dental hygiene program. J Dent Educ 2003; 67: 991-1002. 